\title{
Factors influencing long-term pessary use: reply by the authors
}

\author{
Sarah Friedman • Katherine S. Sandhu • \\ Cuiling Wang • Magdy S. Mikhail • Erika Banks
}

Published online: 2 April 2011

(C) The International Urogynecological Association 2011

Dear Editor,

The publication in April 2010 by Matsubara et al. [1] showed a case series of patients who had complete uterine prolapse and were fitted with ring pessaries. The prolapse in these patients remained resolved even after the pessary removal. Our publication from January 2010 was cited in a subsequent letter to the editor by Matsubara et al. showing the importance of long-term follow-up and identifying factors contributing to successful pessary usage for over 1 year.

We studied 150 women with pelvic organ prolapse using a pessary for over 1 year [2]. Of these 150 women, 115 (77\%) continued using pessaries after 1 year and the

The comment to which this is a reply can be found at http://dx.doi.org/ 10.1007/s00192-011-1394-3

S. Friedman $(\bowtie) \cdot$ K. S. Sandhu $\cdot$ M. S. Mikhail $\cdot$ E. Banks Obstetrics, Gynecology, and Women's Health, Division of Urogynecology and Pelvic Reconstructive Surgery, Albert Einstein College of Medicine, Montefiore Medical Center, 111 East 210th St.,

Bronx, NY, USA

e-mail: obgyninnyc@yahoo.com

\section{E. Banks}

e-mail: ebanks@montefiore.org

\section{Wang}

Epidemiology and Population Health,

Albert Einstein College of Medicine,

Bronx, NY, USA

E. Banks

Albert Einstein Medical School,

1825 Eastchester Road, Room 722,

Bronx, NY 10461, USA remaining 35 (23\%) discontinued usage. The reason for discontinuation was surgery in 25 women and pessary removal without further treatment in the other 10 women. Matsubara et al. wondered whether these 10 patients with pessary removal continued to have prolapse or whether the pessary management was discontinued because the prolapse disappeared or improved. These 10 women discontinued their pessary usage for various reasons: because of ulcerations, discomfort, or annoyance with their pessaries. Though some did show improvement of their prolapse symptoms after removal, we were unable to quantify this. The 12 gynecologists who had managed these 150 women did not all report complete POPQ examinations upon discontinuation of pessary usage.

Our team agrees that a prospective longitudinal study should be performed to determine whether pessary usage can improve prolapse even after discontinuation. Our retrospective chart review did not have an adequate sample size to support or negate Matsubara et al.'s assertion that pessary use may be not only symptomatic relief but also, in some patients, a therapeutic option for uterine prolapse.

\section{References}

1. Matsubara S, Ohki Y (2010) Can ring pessary have a lasting effect to reverse uterine prolapse even after its removal? J Obstet Gynaecol Res 36:459-461

2. Friedman S, Sandhu KS, Wang C, Mikhail MS, Banks E (2010) Factors influencing long-term pessary use. Int Urogynecol J Pelvic Floor Dysfunct 21:673-678. doi:10.1007/s00192-009-1080-x 